\title{
LONGITUDINAL COUPLED-BUNCH INSTABILITY AROUND 1 GHZ AT THE CERN PS BOOSTER
}

\author{
F. Caspers, M. Chanel, H. Schönauer, L. Søby, CERN, CH-1211 Geneva 23, Switzerland \\ M. D’Yachkov, TRIUMF, 4004 Wesbrook Mall, Vancouver, B.C., Canada V6T 2A3
}

\section{Abstract}

The fast-growing "Ring 4" instability occurring at intensities above $6.5 \times 10^{12}$ protons in the top one of the four rings of the CERN PS Booster (PSB) is finally explained by an asymmetry in the 40 vacuum pump manifolds common to all rings. Impedance measurements (by wire method) and numerical calculations show a sharp resonant peak (Q 2000) at $1100 \mathrm{MHz}$ and shunt impedances two times higher for the Ring 4 ports as compared to the other rings. This factor is sufficient to explain that the threshold of the instability falls below the maximum intensity only in Ring 4 . A final, but labor-intensive and expensive, cure consists of inserting short-circuiting sleeves into all 160 beam ports. Results of beam and impedance measurements and the planned cure will be presented and discussed.

\section{INTRODUCTION}

For a number of years, a longitudinal instability occurring solely in Ring 4 above a threshold of $\sim 6.510^{12}$ protons is observed in the PS Booster: During acceleration, around 700 $\mathrm{MeV}$, all bunches develop a fast-growing tail sometimes causing loss of a few percent of the beam. The only coherent signals observed on a wide-band wall current monitor are short bursts visible on all revolution harmonics above $800 \mathrm{MHz}$ up to $1.5 \mathrm{GHz}$. Although there are a number of vacuum tanks and cavities susceptible to drive the instability, they are either common or identical to all four rings. In order to determine whether these cavity-like objects could be the culprits, and whether there is a peculiarity on the level of Ring 4, impedance measurements on spares of the 40 vacuum pump manifolds (vertical connections between the stacked rings) were performed recently.

\section{OBSERVATIONS AND EXPERIMENTS}

Fig. 1 is a waterfall snapshot of the onset of the instability. Minute tails appear simultaneously on the trailing edge of all bunches. Viewed on a slower vertical time scale, it appears obvious that they are the projection of asymmetric tails rotating in phase space at their local synchrotron frequency. Once the phase space asymmetry has smeared out, the bunches show tails on both edges that continue to grow over some tens of milliseconds. Then one sees peaks on a fixed-tuned spectrum analyzer whenever a revolution harmonic sweeps across. Already at intensities just above threshold this growth exceeds the bucket limits and causes loss of a few percent of the circulating beam a few ms later.

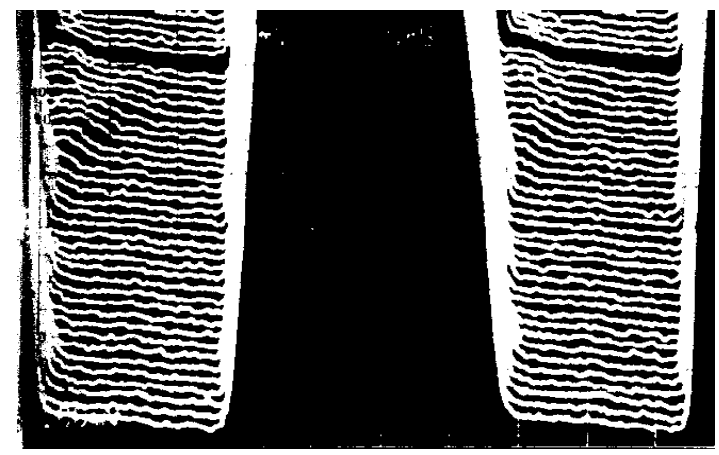

Figure 1: Waterfall display (30 turns between traces, time increases from bottom to top) of the visible onset of the instability. Bunch length is $\approx 65 \mathrm{~ns}$, bunch height about four times the vertical oscilloscope range.

Recently, the instantaneous bunch spectrum above $900 \mathrm{MHz}$ of the instability was measured with a 5 Gsample/s scope with FFT processor and found to be centered around $1 \mathrm{GHz}$. Although the spectrum resembles that of Sacherer's sinusoidal modes, it is wider by a factor six than what would be expected from the bunch length.

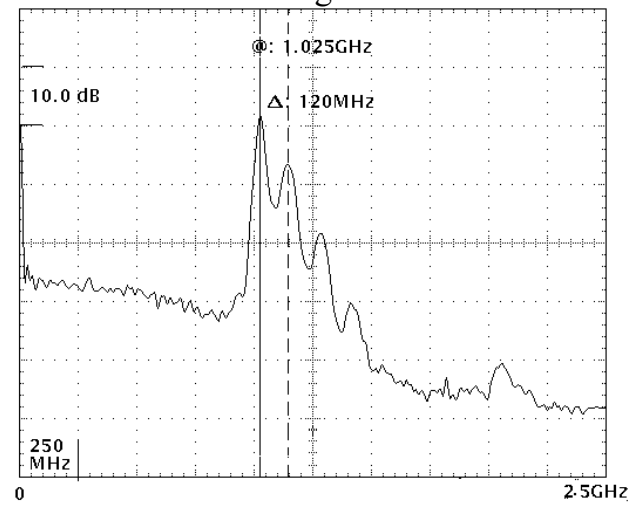

Figure 2: FFT spectrum of the instability. Only the upper half of the spectrum is present, because the wall current monitor signal is filtered through a $900 \mathrm{MHz}$ cut-off highpass to remove the overwhelming stationary bunch structure.

\section{THEORETICAL CONSIDERATIONS}

Before the results of the impedance measurements described below were known, only rough estimates of the impedances needed to drive the beam unstable existed. Straightforward application of the Keil-Schnell-Boussard criterion [1] gives rather low values for the resistive threshold of the order of 25 $\mathrm{k} \Omega$, about the shunt impedance of one single cavity. This criterion holds for wide-band impedances or resonators with $\mathrm{Q}$ $<100$. Using Sacherer's theory of mode coupling [2], recently 
applied to the Fermilab Main Injector by $\mathrm{Ng}$ [3], one finds that for higher $Q$ values $R_{S} / Q$ rather than $R_{S}$ only determines the threshold. Similar result were found also for Gaussian beams [4]. The case of the PSB manifolds with a resonance Q of 2400 is still different: Only one of the many lines of the (coupled-) bunch spectrum can interact with the cavity at one time. This raises the threshold impedance to the order of $1 \mathrm{M} \Omega$. Figure 3 depicts the numerical computation for the Sacherer model of the threshold intensity along the PSB cycle for a resonator shunt impedance of $700 \mathrm{k} \Omega$. The curve refers to the in-phase $(n=0)$ coupled-bunch mode, which has the lowest threshold, but the four other coupled-bunch modes are not very different, contrary to the single-bunch mode whose threshold is one magnitude higher. The coasting beam threshold for a shunt impedance of $30 \mathrm{k} \Omega$ is plotted for comparison. It yields similar threshold intensities to those computed numerically for the resistor-damped manifolds.

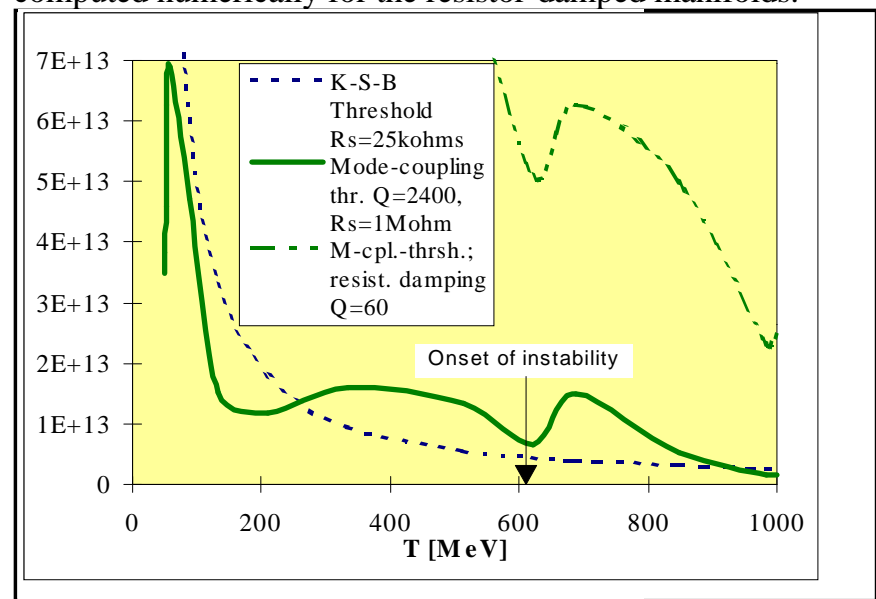

Figure 3: Threshold intensities as function of kinetic energy for K-S-B and mode-coupling criteria. Although the thresholds are comparable, the shunt impedance is $25 \mathrm{k} \Omega$ for $\mathrm{K}-\mathrm{S}-\mathrm{B}$ and $1 \mathrm{M} \Omega$ ( $25 \mathrm{k} \Omega$ for damped resonators) for the mode-coupling theory of bunched beams .

\section{IMPEDANCE MEASUREMENTS: METHOD AND RESULTS}

Of the three spare manifolds made available by the Vacuum Group, \#1and \#2 were slightly different from standard, featuring additional lateral ports (closed here) for insertion of pick-up electrodes. In all beam ports, the beam is shielded from the cavity above and below by strip-like short-circuits. A visual inspection revealed an unexpected feature of all manifolds, namely that the Ring 4 , is shielded only from below, probably because it was felt at design that the proximity of the top wall of the manifold made a proper shortcircuit superfluous. Hence the level 4 resonator volume is indeed of different shape.

Coupling impedances are determined by the standard method of measuring the $\mathrm{S}$ parameters of a wire crossing the test object at the location of the beam, with some precautions to assure a good match at the ports. The impedance of the test object can be calculated from the $S_{21}$ parameter [5]. For the measurements, $500 \mathrm{~mm}$ long standard beam pipes were clamped to the manifold at the ring port under test, and closed with a special flange allowing the soldering of terminating resistors of $270 \Omega$ to the wire under mechanical tension. The resistors were chosen to match the characteristic impedance of $\sim 320 \Omega$ of the wire in the vacuum chamber, to $50 \Omega$. The beam pipe sections were chosen rather long in order not to detune resonances with long evanescent fields into adjacent beam pipes (many cavity modes are beyond waveguide cutoff). In some cases the closed ends of the vacuum pipes were filled with rubber foam to damp the propagating modes above cut-off. Due to the damped propagating modes these measurements are more easily to interpret because the 'baseline' is well-defined.

Another measurement consists of measuring the cavity resonances and their bandwidths by introducing short coupling wires at the gauge ports on the top of the manifold instead of the wire crossing. By checking the $S_{11}$ and $S_{22}$ parameters it was verified that the probes were only weakly coupled to the cavity in order not to reduce the resonator $\mathrm{Q}$ by external loading. From these measurements, one can expect to find the true resonance frequency and $\mathrm{Q}$ values in the absence of the perturbing wire.

\begin{tabular}{|c|c|c|c|c|c|c|c|}
\hline \multirow{2}{*}{\begin{tabular}{|l|} 
Ring \\
port
\end{tabular}} & \multirow{2}{*}{$\begin{array}{c}\begin{array}{c}\text { Mani- } \\
\text { fold }\end{array} \\
\#\end{array}$} & \multirow{2}{*}{\begin{tabular}{|c|} 
Condition \\
s
\end{tabular}} & \multicolumn{5}{|c|}{ Wire Measurement } \\
\hline & & & $f_{\text {res }}$ & $\Delta \mathrm{f}$ & $\mathrm{Q}$ & $\mathrm{R}_{\mathrm{S}}$ & $\mathrm{R}_{\mathrm{S}} / \mathrm{Q}$ \\
\hline & & & $\mathrm{MHz}$ & $\mathrm{MHz}$ & & $\mathrm{k} \Omega$ & $\Omega$ \\
\hline 4 & & & 1100 & 0.44 & 2499 & 62 & 24.8 \\
\hline 3 & & & 1211 & 0.804 & 1506 & 18 & 12.0 \\
\hline 2 & & & 1225 & 1.02 & 1201 & 9.5 & 7.9 \\
\hline \multirow[t]{2}{*}{1} & & & 1220 & 0.596 & 2047 & 19.5 & 9.5 \\
\hline & 1 & & & & & & \\
\hline 4 & & Foam & 1097 & 0.57 & 1925 & 46 & 23.9 \\
\hline 4 & & $\begin{array}{l}\text { Foam + long } \\
\text { damping res. }\end{array}$ & 1097 & 18.2 & 60 & 1.7 & 28.2 \\
\hline 4 & & Foam +2 res. & 1097 & 56.7 & 19 & 0.92 & 47.5 \\
\hline 1 & & Foam & 1225 & 5.25 & 233 & 2.55 & 10.9 \\
\hline 4 & 2 & Foam & 1090 & 4 & 273 & 7.2 & 26.4 \\
\hline 4 & & Foam & 1097 & 0.69 & 1590 & 36 & 22.6 \\
\hline 3 & & Foam & 1239 & 1.1 & 1126 & 13.6 & 12.1 \\
\hline 2 & 3 & Foam & 1237 & 1.8 & 687 & 7.7 & 11.2 \\
\hline 1 & & Foam & 1233 & 1.6 & 771 & 7.8 & 10.1 \\
\hline \multirow[t]{2}{*}{4} & & $\begin{array}{l}\text { Foam+long } \\
\text { damping res. }\end{array}$ & 1083 & 18.5 & 59 & 1.4 & 23.9 \\
\hline & & & \multicolumn{5}{|c|}{ Measurement } \\
\hline \multirow[t]{2}{*}{4} & 3 & $\begin{array}{l}\text { Couplers } \\
\text { into }\end{array}$ & 1055 & 0.44 & 2400 & $54.2^{1)}$ & $22.6^{2}$ \\
\hline & & gauge ports & & \multicolumn{4}{|c|}{$\begin{array}{lll}\text { 1) computed from } R_{S} / Q & \\
\text { 2)taken from } & \text { wire } \\
\text { measurem't }\end{array}$} \\
\hline
\end{tabular}

Table 1: Measured Impedances of PSB Pump Manifolds

The S parameters were measured with a HP 8753C network analyser. Data were dumped via GPIB and Labview software to a Toshiba Laptop. Table 1 compiles the impedances and Q values (determined by measuring or interpolating the $3 \mathrm{~dB}$ 
bandwidths of the resonance peaks). It also includes the impedances of Ring 4 cavities damped by insertion of a ceramic tube coated with a resistive layer. The $R_{S} / Q$ values, being essentially a geometry factor, remain practically unchanged. One notices the outstanding differences between Ring 4 level and the others:

1. Wire measurements show resonance frequencies of 1225 - $1230 \mathrm{MHz}$ for the Ring 1-3 cavities and of $1100 \mathrm{MHz}$ for Ring 4.

2. Equivalent shunt impedances are of the order of $40 \mathrm{k} \Omega$ for Ring 4 and of $8-18 \mathrm{k} \Omega$ for the other rings. Q-values scatter considerably between 700 and 2000 , but

3. $\mathrm{R}_{\mathrm{S}} / \mathrm{Q}$ values are consistently distributed around $24 \Omega$ for Ring 4 and around $11 \Omega$ for the Rings $1-3$.

\section{NUMERICAL COMPUTATIONS}

The results of the measurements were corroborated by numerical finite-differences computation of the wake field of a short bunch (5 ns long), from which coupling impedances can be calculated. The longitudinal impedances found for (a) Ring 1-3 cavities with two short-circuit strips, and (b) the Ring 4 cavity with only one short-circuit strip are published in [6].

Their main features are:

1. The cut-off frequencies (and the resonance frequencies) for the structures are slightly different (frequency of the Ring 4 cavity $=1.0 \mathrm{GHz}$, and of the Ring $1-3$ cavity $=1.15 \mathrm{GHz}$ ).

2. Impedances at resonance are significantly different; it looks as though $\mathrm{R}_{\mathrm{S}} / \mathrm{Q}$ (which should be proportional to the integral of $\int \mathrm{R}(\omega) \mathrm{d} \omega$ of the Ring 4 cavity) is approx. 2.5 times higher than the $\mathrm{R}_{\mathrm{S}} / \mathrm{Q}$ for the Ring 1-3 cavity.

3. There is also a big difference in transverse impedances of the structures.

\section{POSSIBLE CURES}

An efficient measure to reduce the coupling impedance of the manifolds is the insertion of flexible perforated sleeves into all beam ports. As the manifolds support all the vacuum pumping system, this is a laborious operation which is envisaged for the next PSB shutdown. Although the insertion of damping resistors is much easier, it is not proposed as it is not feasible for the rings 1-3 and the predictable instability threshold is lower compared to the one obtained by shortcircuiting sleeves.

\section{CONCLUSIONS}

Both wire measurements of longitudinal coupling impedance and wake field calculations agree that the pump manifolds act as individual high-Q resonators for each ring, where Ring 4 stands out by a factor $\sim 2.5$ w.r.t. the other rings both in $R_{S}$ $(\sim 50 \mathrm{k} \Omega)$ and $\mathrm{R}_{\mathrm{S}} / \mathrm{Q}(\sim 25)$. $\mathrm{Q}$ values vary over the measurements but can reach values $>2000$.

Resonance frequencies (measured $1.055 \mathrm{GHz}$ and calculated 1 $\mathrm{GHz}$ ) for the Ring 4 cavities are also lower by about 130 $\mathrm{MHz}$ with respect to the other levels. There is a difference of $50 \mathrm{MHz}$ between calculations and wire measurements. These results suggest that the pump manifolds might indeed be the driving impedance of the 'Ring 4 Instability', distinguishing this ring from the others.

Straightforward application of the Keil-Schnell-Boussard criterion for stability yields for the observed threshold intensity an impedance of the order of $25 \mathrm{k} \Omega$. This would fit the measurements if there were only one manifold in the ring or if the resonances of the present 40 manifolds do not overlap.

Application of mode-coupling theory for extremely narrow resonances, which interact with only one spectral line of a mode, yields impedance thresholds of the order of $1 \mathrm{M} \Omega$, which is possible only if the resonance curves of the manifolds strongly overlap. From the resonance frequencies measured on three manifolds (two coincide, while the third is $6 \mathrm{MHz}$ lower), this seems not impossible but it is difficult to guess the whole distribution. A scatter of the resonance frequencies may also explain the intriguing fact, that adding 10 manifolds in 1993 has changed neither the threshold of the instability nor the ensuing blow-up. In spite of the open questions, the evidence is convincing enough to justify the cure of short-circuiting all ports by flexible sleeves. In the case of a cure by systematic insertion of damping resistors, individual resonant impedances will certainly overlap and their superposition may dangerously approach the coastingbeam criterion.

\section{ACKNOWLEDGEMENTS}

We would like to thank the PS Section of the Vacuum Group for their efficient help in the preparation of the measurement.

\section{REFERENCES}

[1] D. Boussard, 'Observation of microwave longitudinal instabilities in the CPS', CERN/LabII/RF/75-2 (1975); E. Keil, W.Schnell, 'Concerning longitudinal stability in the ISR', CERN/ISR/TH/RF/ 69-48 (1969) .

[2] F. J. Sacherer, 'Bunch lengthening and the microwave instability', Trans. IEEE Nucl. Sc. NS-24, 1393 (1977), and CERN/PS/BR 77-6 (1977).

[3] K. Y. Ng, 'Mode-coupling instability and bunch lengthening in proton machines', Proc. of the 1995 Part. Acc. Conf. 2977 (1995), Dallas 1995.

[4] T. Bohl, T. P. Linnecar, E. Shaposhnikova, 'Measuring the resonance structure of accelerator impedance with single bunches' CERN-SL-96-62 RF (1996), and this Conference.

[5] F. Caspers in 'RF Engineering for Particle Accelerators', CERN Accelerator School Proceedings CERN 92-03, p. 128 .

[6] F. Caspers, H. Schönauer, L. Soby, 'Impedance measurements on Booster manifolds', CERN PS/HI/Note 96-15 (1996). 